\title{
85662 - AÇÃO DAS VITAMINAS ANTIOXIDANTES A, C E E NO TRATAMENTO DO CÂNCER DE MAMA
}

\author{
Pôster - Geriatria
}

Adriana Eckhardt, Tereza Cristina Blasi e Natielen Jacques Schuch / Eckhardt Adriana; Blasi,TC e Schuch, NJ / UFN

Introdução: $\mathrm{O}$ câncer de mama é um dos mais difundidos nas mídias por ser o tipo que mais afeta a população feminina. Vários são os fatores de risco para o aparecimento do câncer de mama, dentre eles o sedentarismo, dieta, obesidade e os fatores dietéticos. $\mathrm{O}$ estresse oxidativo está relacionado não somente à toxicidade do tratamento da doença, mas também à própria etiologia do câncer. Sendo assim, os antioxidantes podem auxiliar tanto na prevenção do câncer de mama quanto no seu tratamento. Objetivo: O presente artigo tem por objetivo revisar os principais estudos relacionados ao papel dos antioxidantes das vitaminas A C e E no tratamento do câncer de mama. Metodologia: revisão de literatura, com buscas de artigos científicos nas bases de dados ScienceDirect, PubMed, Web of Science e na biblioteca eletrônica SciELO, a fim de identificar os trabalhos publicados e disponíveis até março de 2019, utilizando os termos indexadores: antioxidantes AND vitamina A AND vitamina C AND vitamina E AND câncer de mama. Resultados: Vários estudos realizados com mulheres em tratamento quimioterápico demonstraram efeitos positivos com a suplementação e/ou ingestão dietética desses nutrientes, emergindo assim, sua ação anticarcinogênica e antioxidante. Sendo assim, é de grande importância a orientação adequada desses nutrientes, afim de minimizar os danos e os riscos de morte causados pela doença. Conclusão: Diante deste contexto, a quimioprevenção através dessas vitaminas emerge como um importante instrumento de prevenção e controle de câncer de mama, sugerindo mecanismos de ação anticarcinogênicos e antioxidantes. 